\title{
Nr. 8 GOÄ nicht vergessen!
}

Die Nr. 8 GOÄ setzt eine Untersuchung der Haut, der sichtbaren Schleimhäute, der Brust- und Bauchorgane, der Stütz- und Bewegungsorgane sowie eine orientierende neurologische Untersuchung voraus. Übersehen wird häufig, dass nur eine „Untersuchung“ gefordert ist - und nicht wie z.B. bei den Nrn. 6 und 7 eine „vollständige“ oder wie bei der $\mathrm{Nr}$. 800 eine „eingehende“ Untersuchung.

\section{MMW-KOMMENTAR}

Konkret bedeutet dies, dass man zwar alle genannten Bereiche des Körpers untersucht haben muss - es heißt ja auch „Ganzkörper- status" -, aber nur in der im Einzelfall medizinisch notwendigen Intensität. Dass für den Ganzkörperstatus lediglich eine "geringere Gründlichkeit" der Untersuchung verlangt ist, wird allein schon daraus erkenntlich, dass die Leistung nur mit 260 Punkten bewertet ist. Wäre eine Gründlichkeit impliziert, wie sie z. B. die Leistungsbeschreibung der Nr. 7 für Haut und Bewegungsapparat fordert, entspräche allein das schon einem Wert von 320 Punkten. Hinzu käme die orientierende neurologische Untersuchung, entsprechend der Nr. 5 und damit zusammen eine kalkulatorische Bewertung mit 400 Punkten. So ist es aber eben nicht.

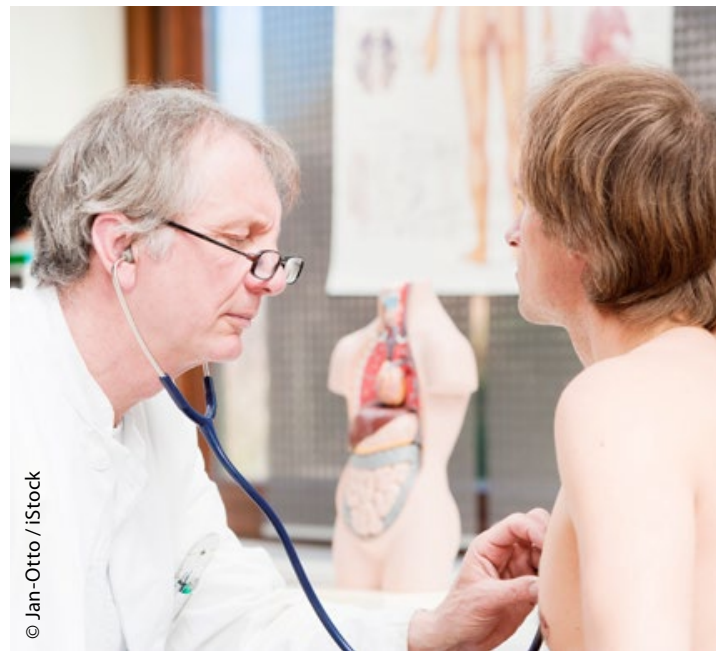

Der Ganzkörperstatus ist eine Untersuchung von "geringer Gründlichkeit"

\section{Nr. 03242 EBM kann man „auf Lücke“ abrechnen}

_ Die Nr. 03242 EBM steht für die Abrechnung von Testverfahren bei Demenzverdacht. Obligater Leistungsinhalt ist die Beurteilung von Hirnleistungsstörungen mittels standardisierter Testverfahren bei Patienten mit Demenzverdacht. Die Leistung kann je Test, aber nur bis zu dreimal im Quartal berechnet werden und ist mit 1,98 Euro bewertet.

\section{MMW-KOMMENTAR}

Nicht selten äußern ältere Patienten gegenüber ihrem Hausarzt den Verdacht, dass sie eine Demenz entwickeln, weil ihr Kurzzeitgedächtnis nachlässt. Diese Patienten kann man mit dem Ergebnis solcher Testverfahren in der Regel beruhigen. Da die Legende der Nr. nur einen Demenzverdacht fordert, kann sie auch berechnet werden, wenn sich der Verdacht nicht bestätigt. Die Durchführung kann kom- plett an Praxispersonal delegiert werden, weshalb es auch keine Zeitvorgabe für die Wirtschaftlichkeitsprüfung gibt. Die Nr. 03242 ist im Behandlungsfall nicht neben der Nr. 03360 berechnungsfähig. Da letztere aber praktisch nur jedes zweite Quartal berechnet werden kann, wäre ein Ansatz der Nr. 03242 auch bei Patienten, die das 70. Lebensjahr überschritten haben, zumindest "auf Lücke" möglich.

\section{Kein Fortbildungsregress aus heiterem Himmel!}

_ Wer innerhalb von fünf Jahren nicht 250 Fortbildungspunkte nachweist, dem drohen Honorarkürzungen von bis $\mathrm{zu}$ 25\%. Die KV muss zuvor aber eindeutig auf diese Gefahr hinweisen.

\section{MMW-KOMMENTAR}

Das hat gerade erst das Landessozialgericht Nordrhein-Westfalen bestätigt (Az.: L 11 KA 106/12). Die KV hatte einem Arzt wegen feh- lender Fortbildungsnachweise das Honorar für das 2. Quartal 2011 um rund 10.000 Euro gekürzt. Der Sachbearbeiter habe den Arzt im Juni 2010 darauf hingewiesen, dass er für den Nachweis nur noch bis zum April 2011 Zeit habe. Dieser blieb trotzdem aus.

Der Arzt legte Widerspruch ein und wies darauf hin, dass er sich sehr wohl ausreichend fortgebildet habe. Es könne ihm nur vorgeworfen werden, dass er die Nachweise nicht rechtzeitig übersandt habe. Darauf hätte ihn die KV mindestens drei Monate vor Ablauf der Frist hinweisen müssen. Das KV-Schreiben von Juni 2010 habe er nicht erhalten. Tatsächlich konnte die KV die Zustellung des Schreibens nicht nachweisen - wozu sie laut dem Gericht aber verpflichtet sei. Es reiche nicht aus, wenn im Verbandsorgan allgemein auf die Fortbildungspflicht der Ärzte hingewiesen werde. 